\title{
علاقة همارسة إدارة الجودة الشاملة على الرضا الوظيفي في الفنادق الأردنية
}

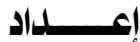 \\ د. سلامة سويلم النو|فعة \\ الأستاذ المسـاعد - جامعة البلقاء التطبيقية
}

مجلة بحوث التربية النوعية - جامعة المنصورة

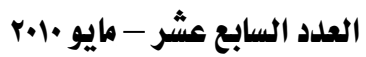




\section{علاقة همارسة إدارة الجودة الشاهلة على الرضا الوظيفي في الفنادق الأردنية \\ إعـــاد}

د ـ سامة سـويلم النوافعة

صناl|

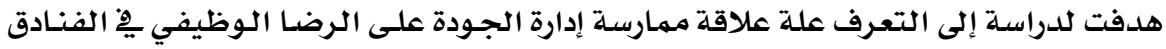

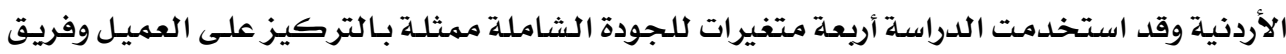

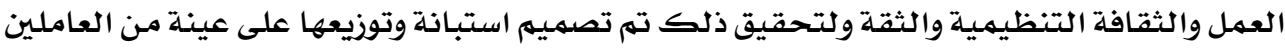

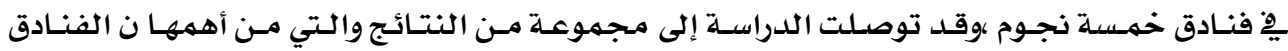

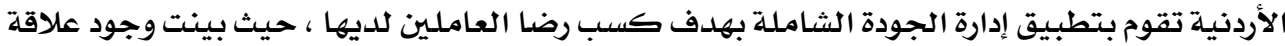

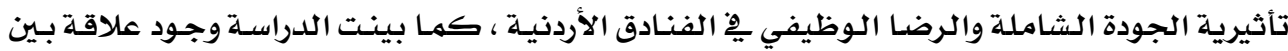

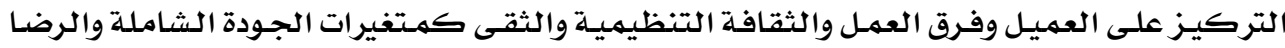

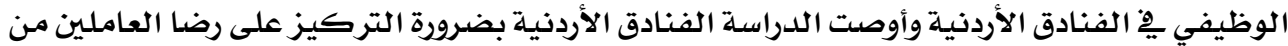

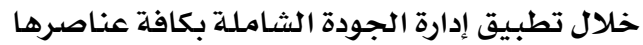
الكلمات الدالة : إدارة الجودة الشاملة، الرضا الوظيفي ، الفنادق الأردنية 
Abstract

The study aimed to investigate the relation of total quality management with job satisfaction in Jordan hotels. Four principals of total quality management variables were selected represented by (customer focusing, teamwork, organizational culture and trust). Self administrated questionnaire was designed and distributed over the research sample, totaling 150 . The study concluded that Jordan hotels implement total quality management for the purpose of obtaining staff satisfaction. It also found relation between TQM and job satisfaction. The study recommended that Jordan hotels should focus on job satisfaction through implementation all elements of total quality management

Keywords: Total quality Management, Job Satisfaction, Jordan Hotels 


\title{
علاقة همارسة إدارة الجودة الشاهلة على الرضا الوظيفي في الفنادق الأردنية
}

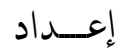 \\ د ـ سلامة سويلم النوافعة
}

هقدهة

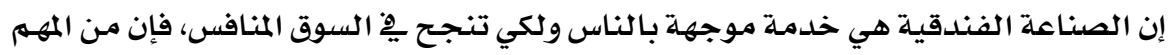

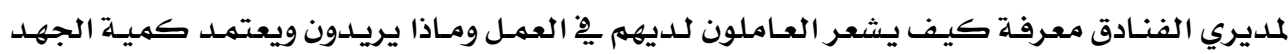

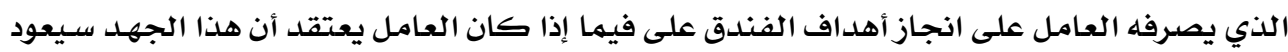
إلى تلبيلة حاجاته ورغباته.

أعطـى البـاحثون والاختــصاصيون الرضـا الـوظيفي أهميـة كـبيرة لـا يـشكله مــن أهميـة

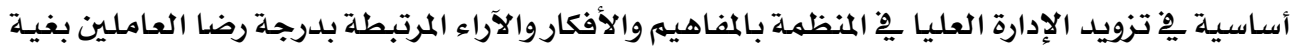

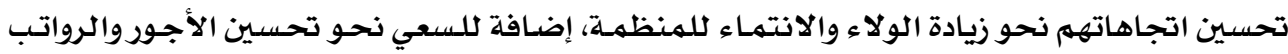

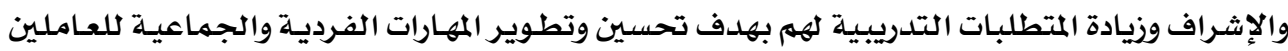

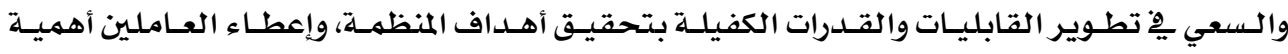

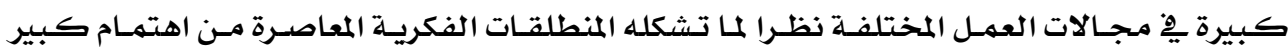

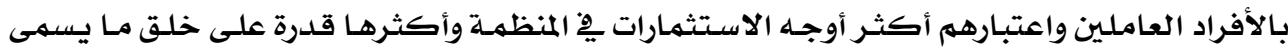
بمنظمـة المعرفة بهدف تحسين الإنتاج من الناحيتين الكميـة والنوعية.

حاولت دراسات سابقة ربط إدارة الجودة الشاملة ورضا العاملين كانت نتائجها محسلدودة مـن

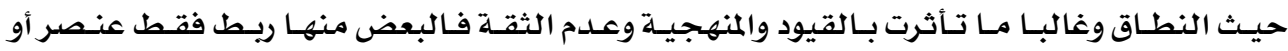

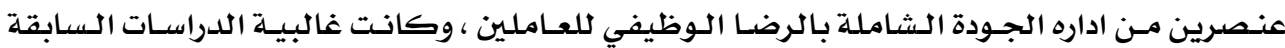

نظريـة فقط وقدمت أدله قليلة لتدعيه استنتاجاتها (Chapman and Al- Khawaldeh , 2003)

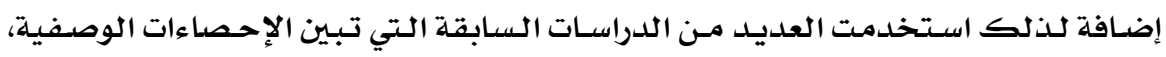

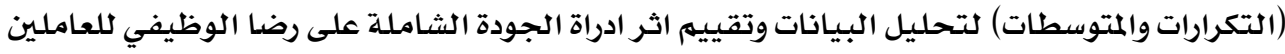

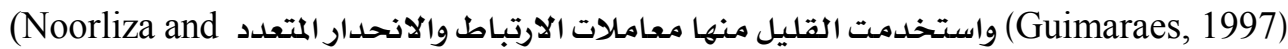
.Zainal , 2000)

\section{مشكلة الدراسة - ماسة}

تعتمد الصناعة الفندقية بشكل كبير جدا على العنصـر البشري يِّ تقدم خـدماتها وتعتمـد

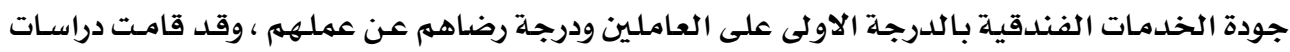

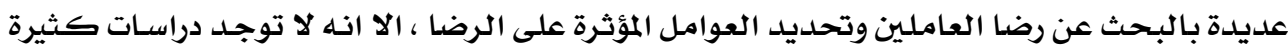


تتتـاول اثر إدارة الجـودة الشـاملـة على الرضـا العـاملين الوظيفي ومـن هنـا فـان هـذه الدراسـة تستخدلم

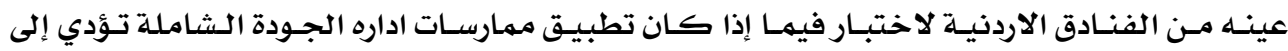

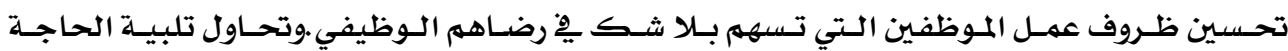

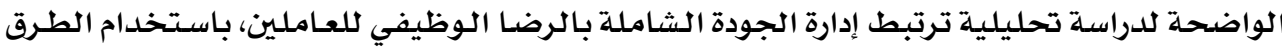
الإحصائية المناسبـة يِّ الفنادق الأردنية وتستخدم هذه الدراسة بعض العناصـر الرئيسية لإدارة الجـودة

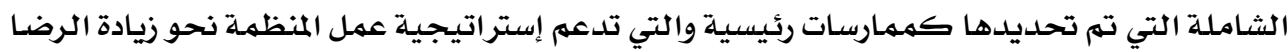

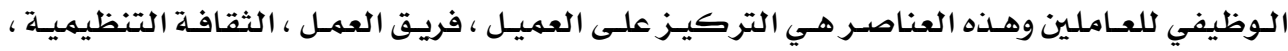
والثقة التتظيمية. أسئلة الدراسة

• ما هي ممارسات إدارة الجودة الشاملة الضروريـة التي يمكن تطوريها لتكون دليلا فاعلا ِِّ قياس

$$
\text { الرضا الوظيفي للعاملين }
$$

• هل تؤثر ادراكات العاملين لممارسات إدارة الجودة الشاملـة على رضاهم الوظيفي ل

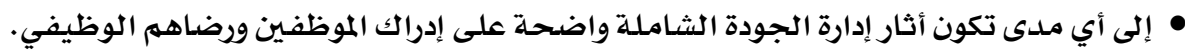

وتتبع أهميـة الدراسـة بشكل خاص لأنها تسعى لكثف درجـة التـأثير التي بها يفرض تطبيق

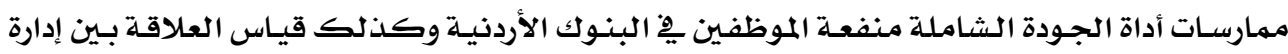

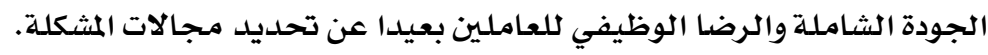

\section{أهداف الدراسة الجوده}

تهدف هذه الدراسة للتعرف على اثر مبادئ إدارة الجـودة الشاملة ِيْ الرضـا الـوظيفي، وثانيا

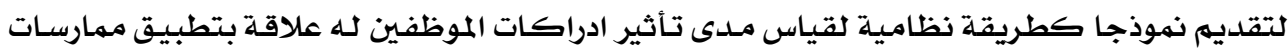
ادراة الجودة الشاملة على الرضا الوظيفي والتي أصبـحت عنصـرا كـاملا مـن أي منظمـة عنـدما يكـون

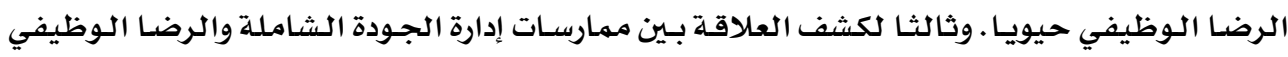

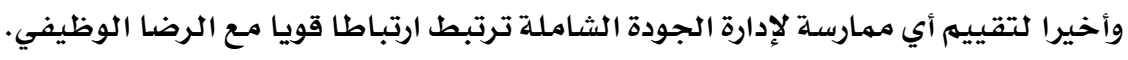

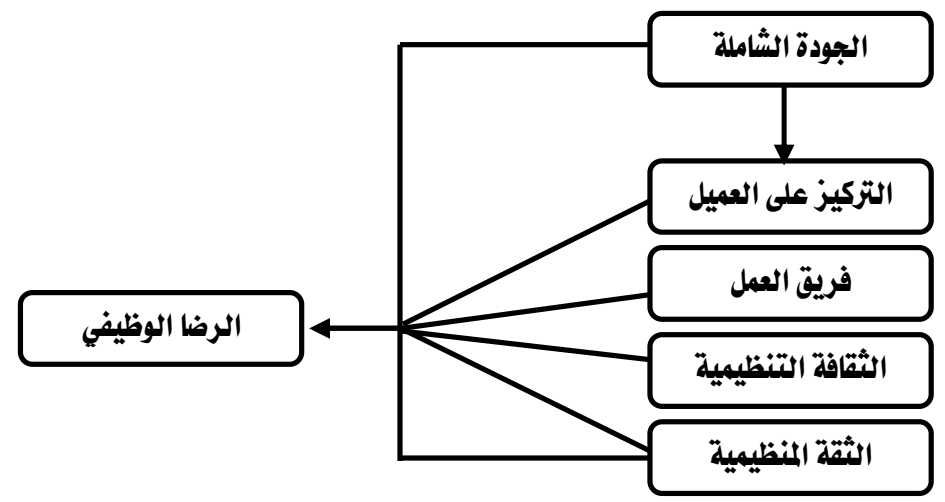




\section{فرضيات الدراسة}

الفرضية الرئيسية

توجد علاقة بين الجودة الشاملة يِ الفنادق والرضسا الوظيفي

ويتفرع عن هذه الفرضيـة الفرضيات التالية:

• الفرضية الفرعية الأولى:

توجد علاقة بين التركيز على العميل كأحد متغيرات الجودة الشاملة والرضا الوظيفي ِِِ

الفنادق الأردنية

• الفرضية الفرعية الثانية :

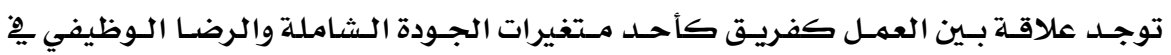

الفنادق الأردنية

• الفرضية الفرعية الثالثة:

توجد علاقة بـين الثقافة التنظيميـة كأحسد مـتغيرات الجـودة الشاملـة والرضـا الوظيفي ِِِ

$$
\text { الفنادق الأردنية }
$$

• الفرضية الفرعية الرابعة:

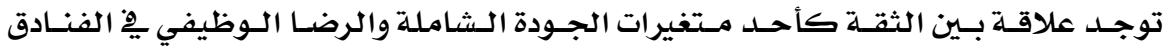

الأردنية

\section{أدبيات الدراسة}

تم تعريف الرضا الوظيفي وقياسـه بأبعاد متعددة فقد تم تعريف الرضا الوظيفي كوظيفـة

للعلاقات المدركة وما يريد الفرد من وظيفته وما يحصل عليه من وظيفته وقد قامت دراسـات متعـددة

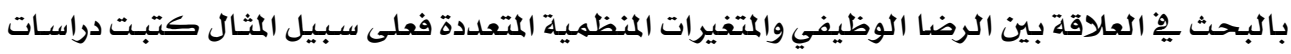

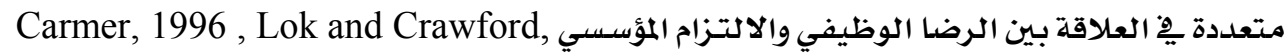
المظف 1999, Currvan, 1999,

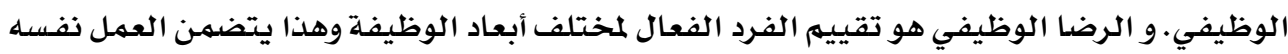

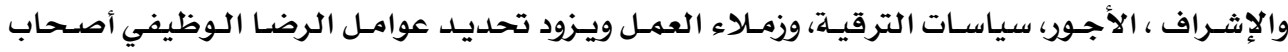

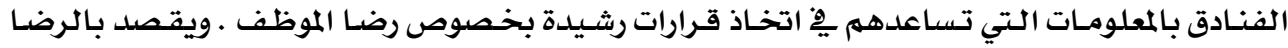

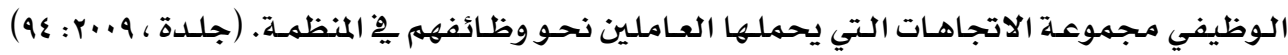

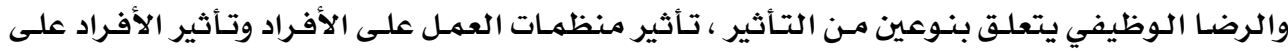

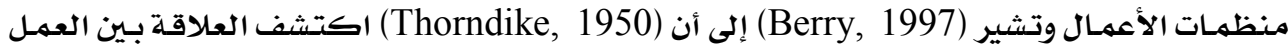

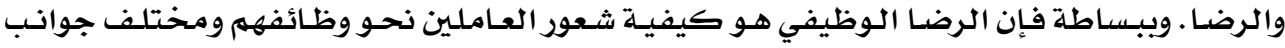

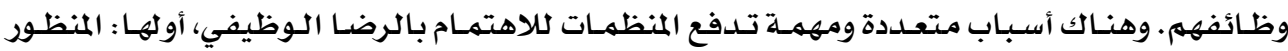




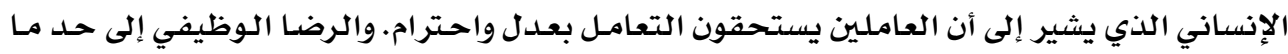

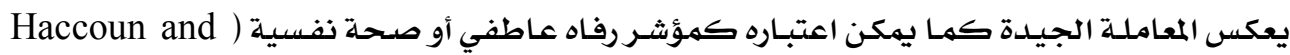

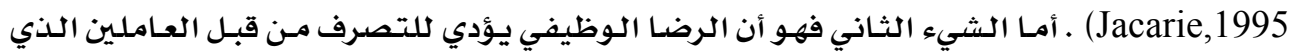

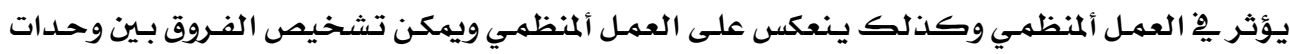

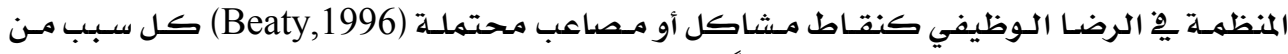

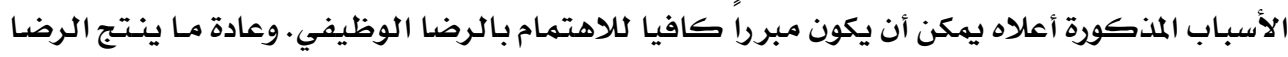

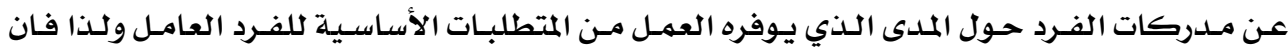

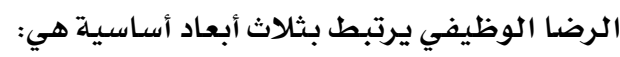

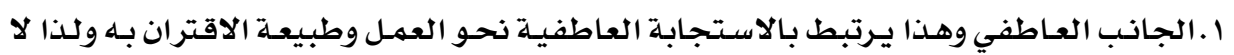

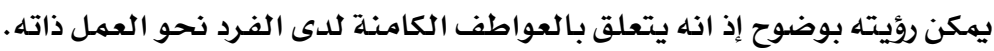

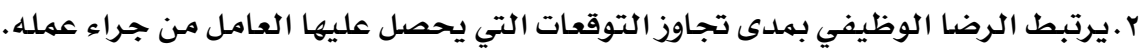

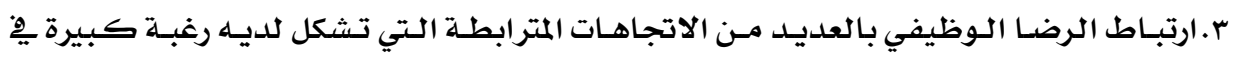

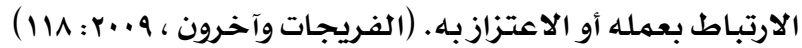

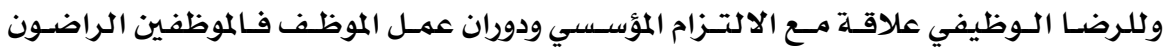

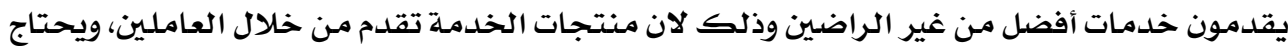

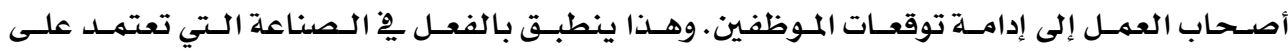
التفاعلات البشرية (Spinelli and Canavos, 2000) مثل الصناعة الفندقية ويشير ( - McNeese

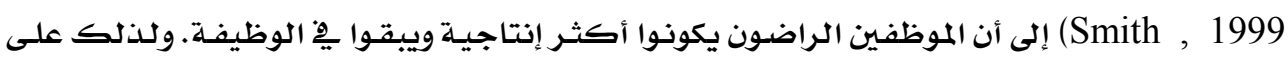

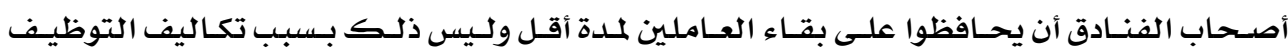

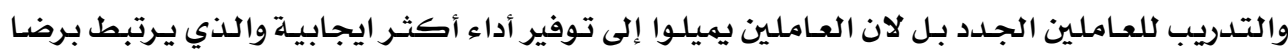

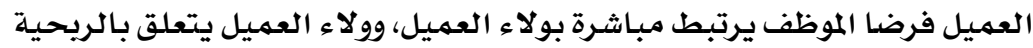

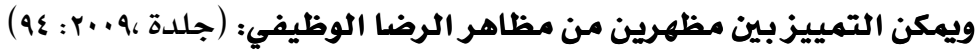

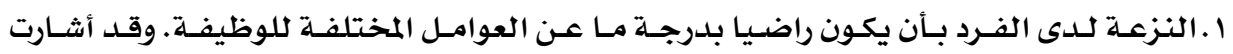

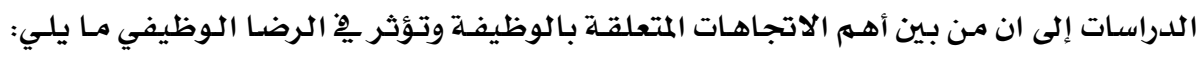

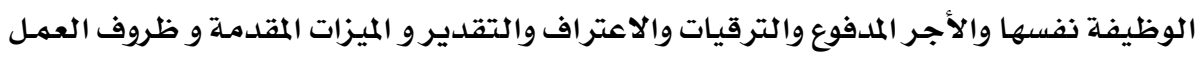

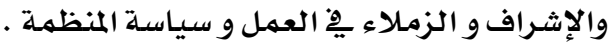

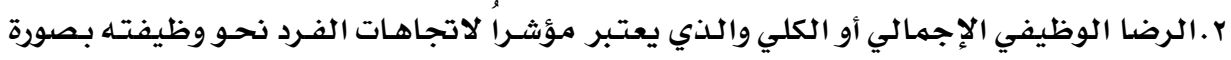

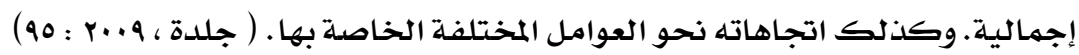

عوامل تسهم في الرضا الوظيفي

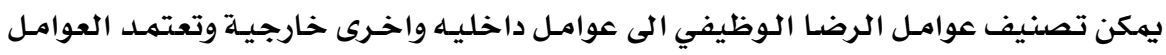

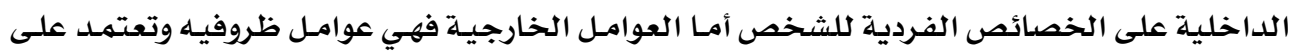

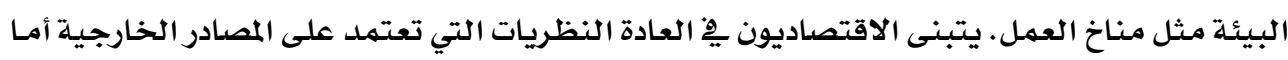




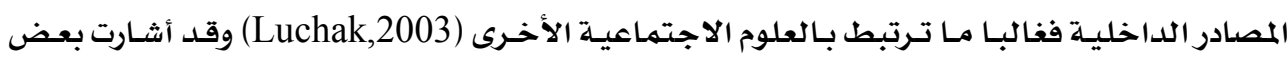

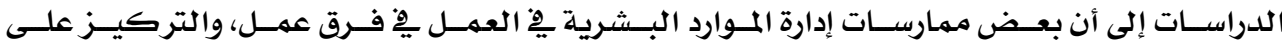

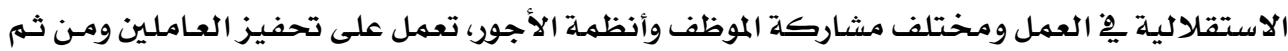

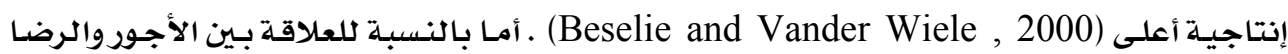

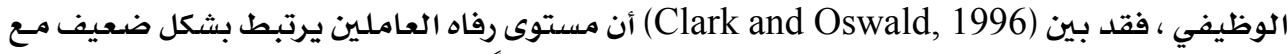

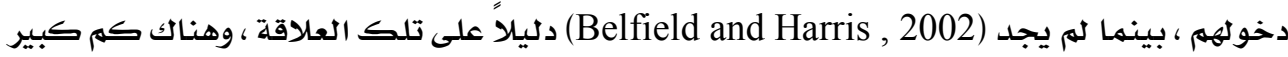

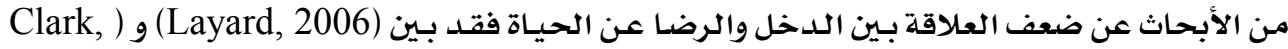

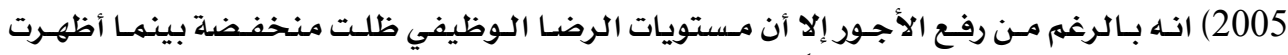

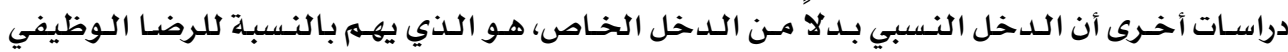

(Clark and Oswald, 1996)

وقد قسم (Kelly and Lee, 2009) المؤثرات على الرضا الوظيفي إلى عوامل داخلية تتعلق

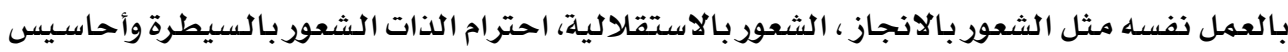

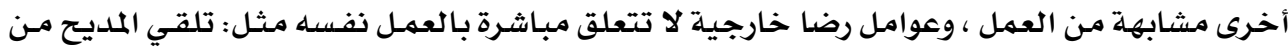

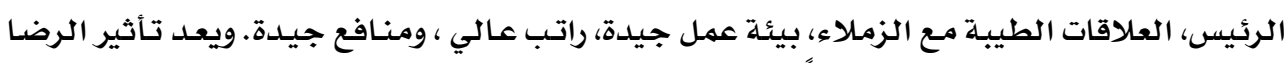

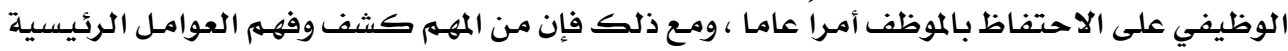

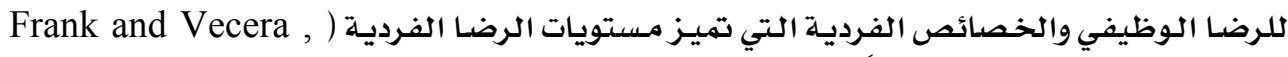

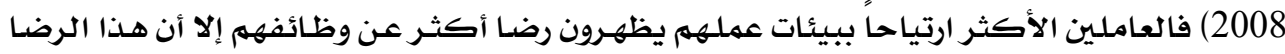

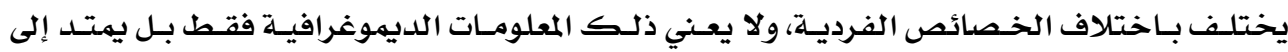

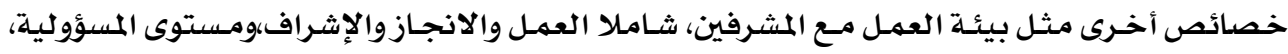
والمنظمة التي يعمل بها، ونوع العمل، ومدة العمل، والتعويض (Kacel et al , 2005)

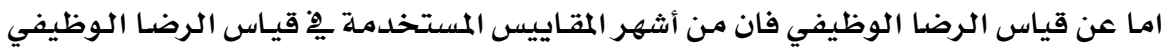

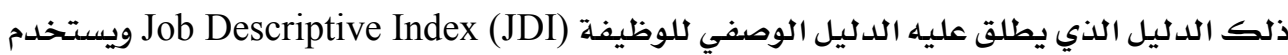

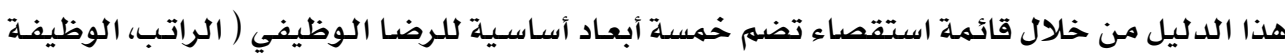

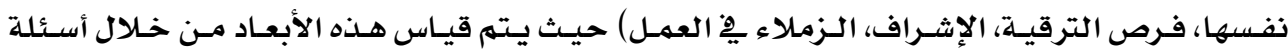

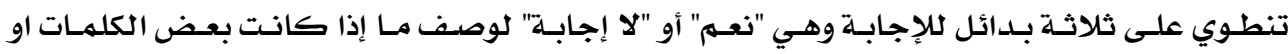

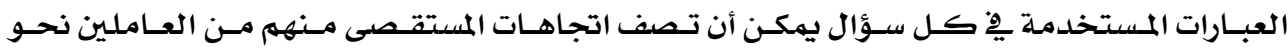

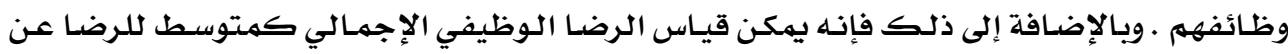

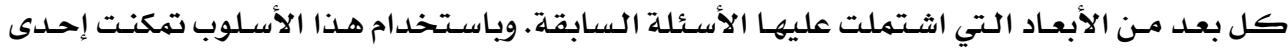

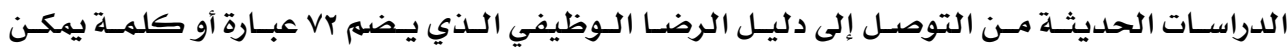

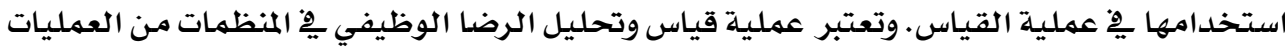

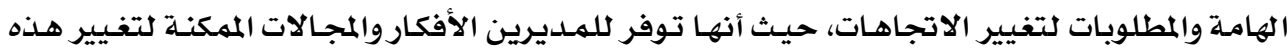

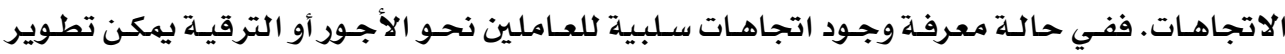

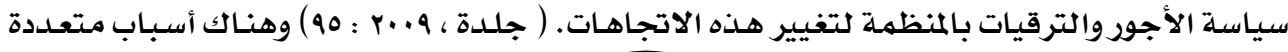




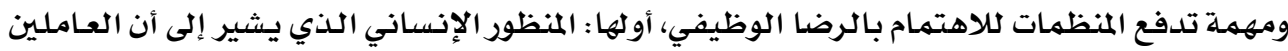

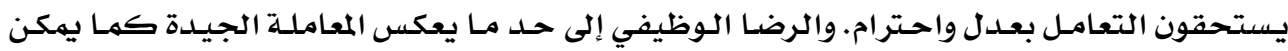
اعتباره كمؤشر رفاه عاطفي أو صحة نفسية (Haccoun and Jacarie,1995) .

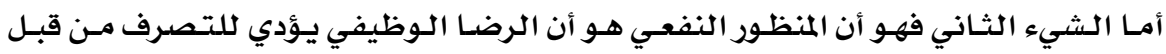

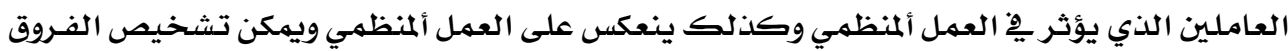

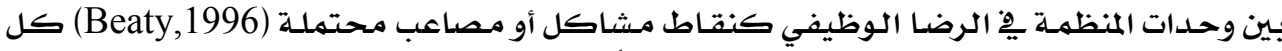
سبب من الأسباب المذكورة أعلاه يمكن أن يكون مبرراً كافيا للاهتمام بالرضا الوفا الوظيفي.

\section{مفهوم إدارة الجودة الشاملة}

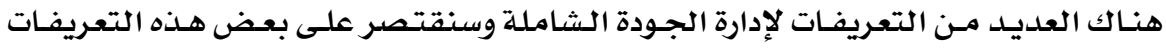

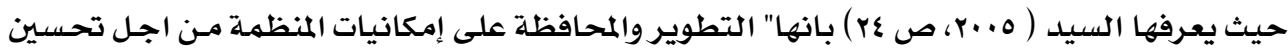

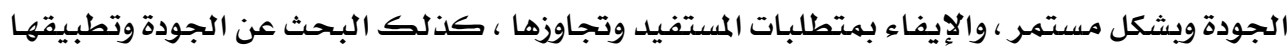

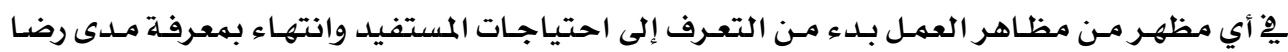

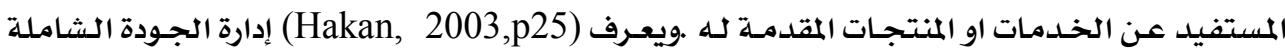

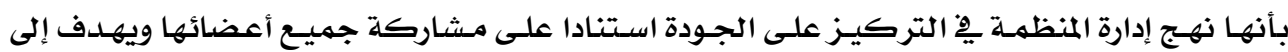

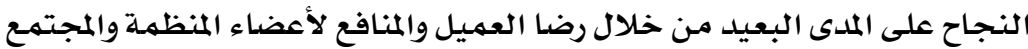

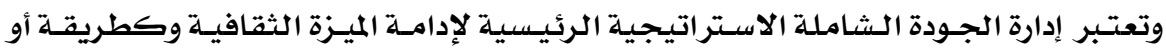

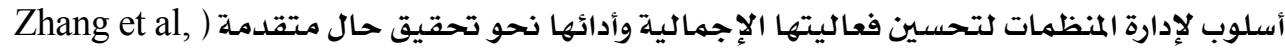

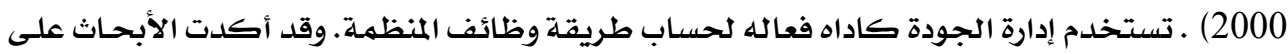

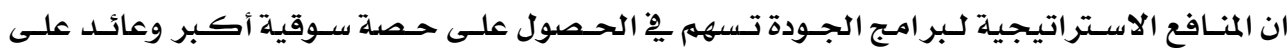

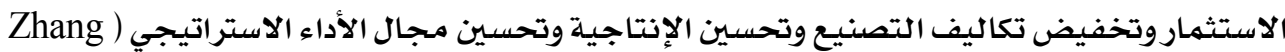
(et al , 2000

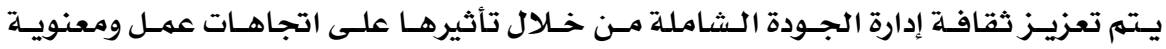

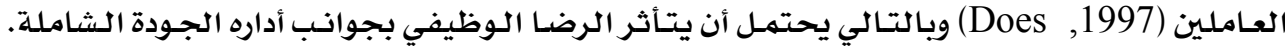

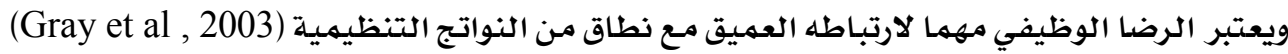

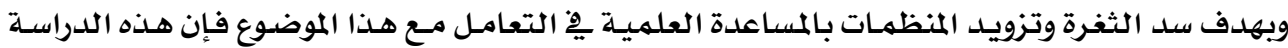

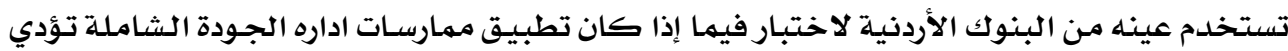

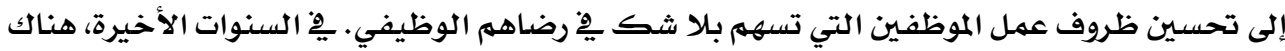

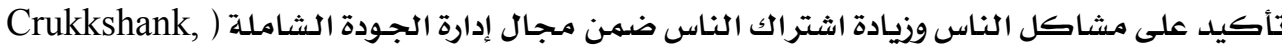

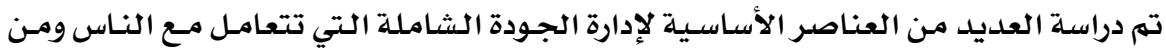

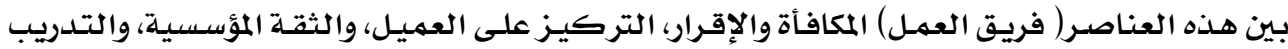




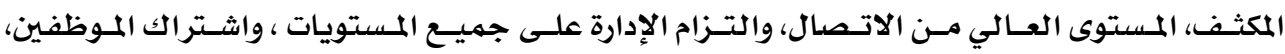
والتمكين و الثقافة التتظيمية (Noorliza and Zainal, 2000 , Ockland and Ockland, 2001) وقد وجدت الدراسـات أن تأثير تحسـين جـودة حيـاه العهل تعمل على تقليل مـن دوران العهـل

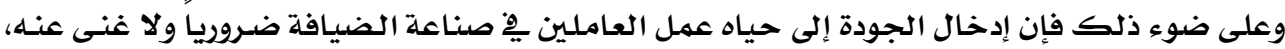

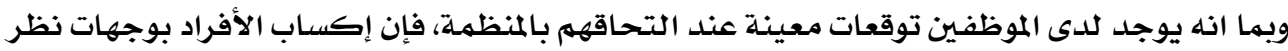

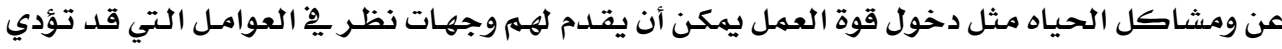
إلى نواتج سلبيية(Cleveland et al 2007)

$$
\text { وتركز إدارة الجودة الشاملة على ما يلي ( سملالي ، ب... ) }
$$

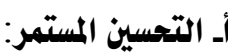

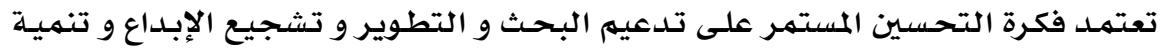

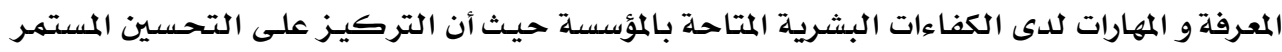

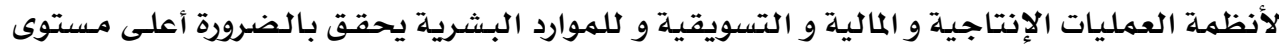

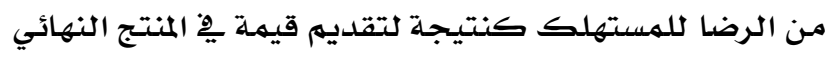

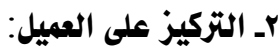

أصببح العميل محسل اهتهـام متزايـد مـن كافـة المنظمـات لاقتصادية فالاحتفـاظ بـالموقف

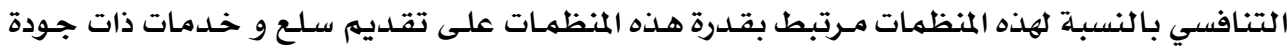
تلائهم أذواق العملاء و تلبي احتياجـاتهم المحلددة أو الشاملة.

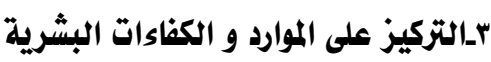

يشكل العنصر البشري أحد أهم العناصر الانتاجية ِِِ منظمـات الأعمال وخاصـة المنظمات

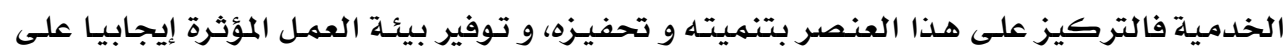

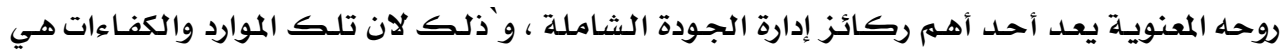

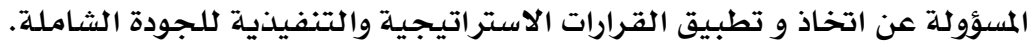

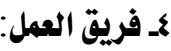

ان اشتراك العاملين يِّ عمل جماعي يساعد على زيادة الولاء والانتماء للمؤسسة وأهدافها

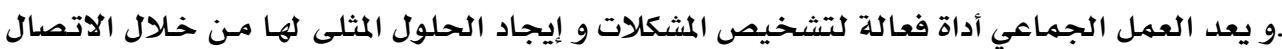
المباشر بين مختلف العاملين و الاحتكاك المستهمر بينهم

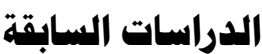

دراسـة (Keng et al , 2007). التي هـدفت الدراسـة إلى بحثث ادراكـات الموظفين لممارسـات

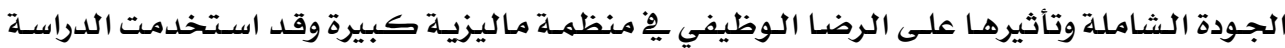

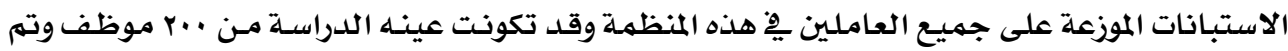




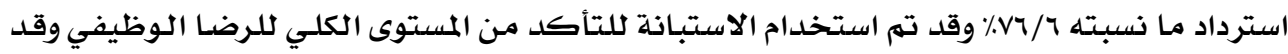

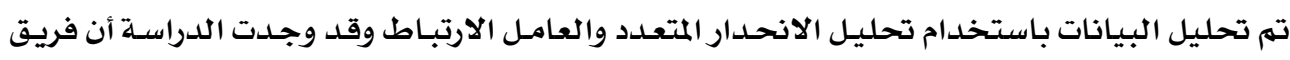

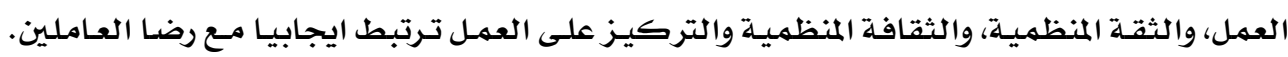

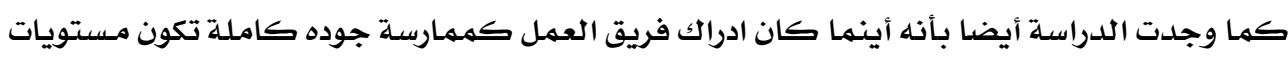

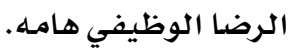

بينت دراسـة (Alina and Rob, 2006) التي هدفت إلى البحث بِّ العلاقـة بـين ممارسـات

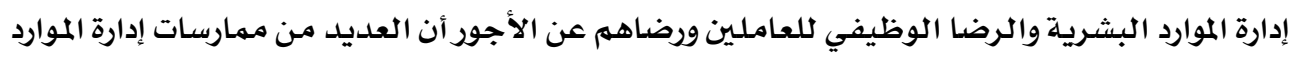

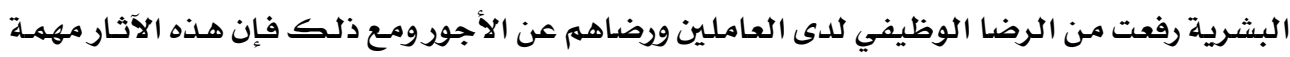

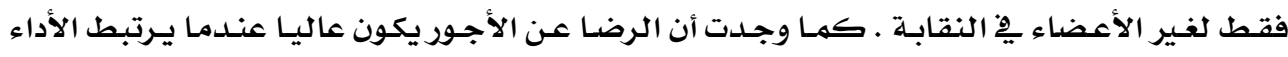
بالأجورونظم المكافأة

بحثت دراسـة (Alf and Bassem , 2003) يُّ العلاقات بين الرضـا الوظيفي ، وجوانب

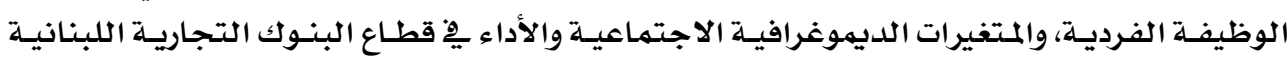

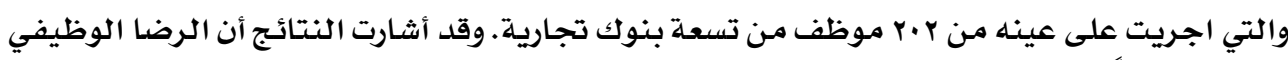

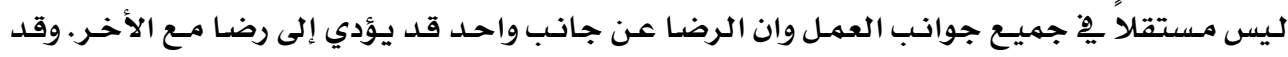

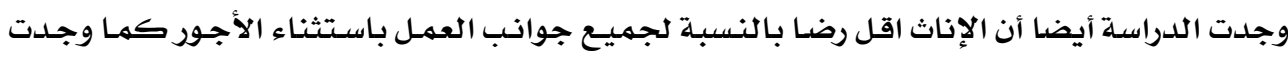

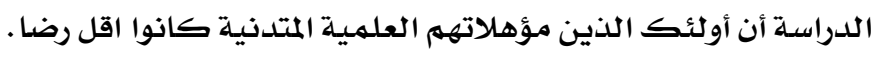

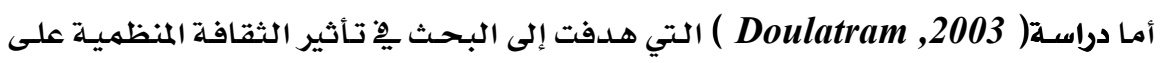

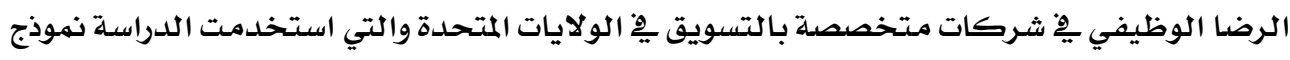

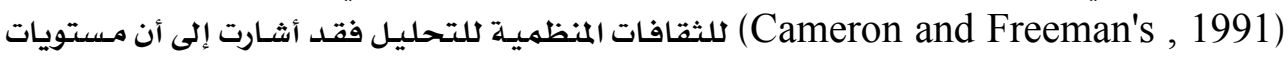
الرضا الوظيفي تختلف باختلاف ثقافة المنظمة .

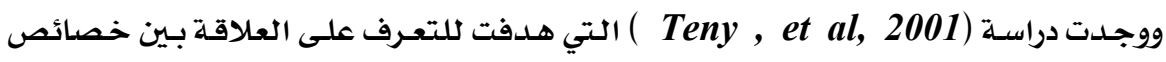

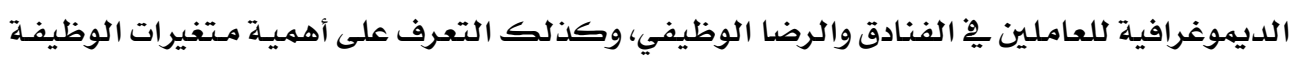

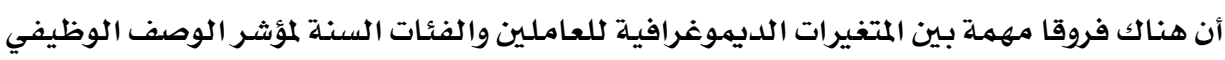

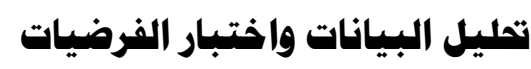

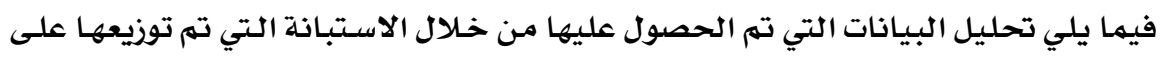

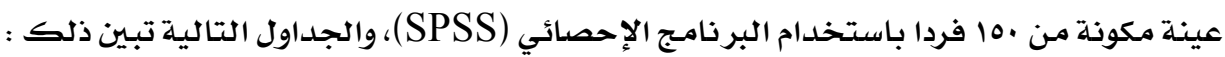




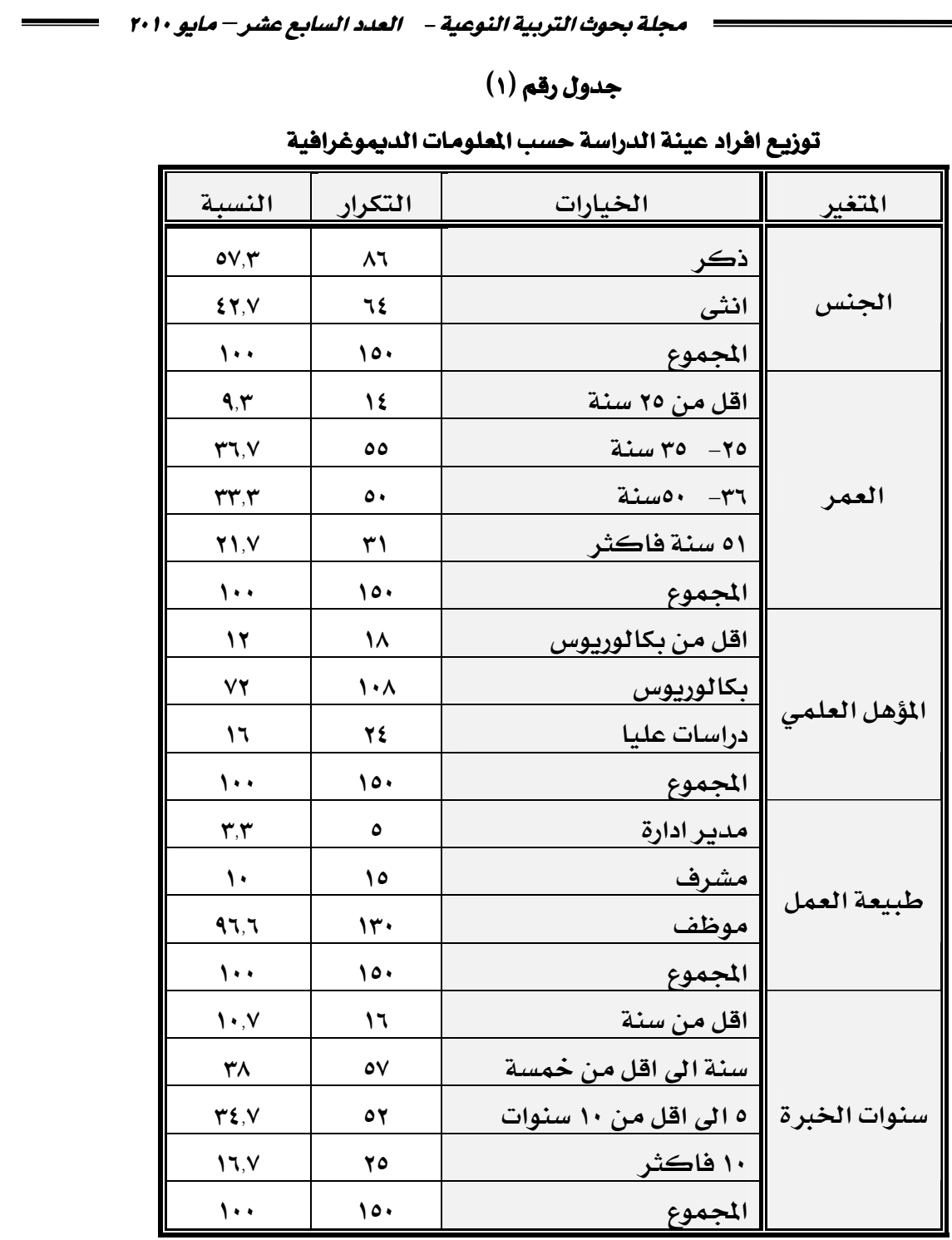


جدول رقم (ץ)

المتوسطات والانحرافات المعيارية لفقرات الاستبيان ككل

\begin{tabular}{|c|c|c|c|}
\hline الانحراف & المتوسط الحسابى & 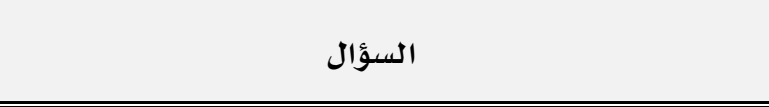 & رقمر \\
\hline & & اولا : التركيز على العميل & \\
\hline $1,|r|$ & $\mathrm{r}, \Lambda \mathrm{V}$ & يعالج الفندق شكاوي الضيف بسرعة & 1 \\
\hline$\cdot, \wedge \mathrm{VV}$ & $\varepsilon, r v$ & يشكل رضـا الضيف اواوية للفندق & r \\
\hline $1,19 \mathrm{~V}$ & $\varepsilon, 1$ & يقوم الفندق بقياس رضا الضيف مـن فترة لاخرى & r \\
\hline $1, \cdot 19$ & $\varepsilon, \pi$ & يولي الفندق اهتماما متزايدا بالضيف & $\varepsilon$ \\
\hline $1, \cdot r_{0}$ & $\varepsilon, 0 \mathrm{~V}$ & يحرص الفندق على تقديم خلدمات تتفق وتوقعات الضيف & 0 \\
\hline \multirow[t]{2}{*}{1,91} & $\xi, r V$ & يركز الفندق على تحسين الخدمات المقدمـة للضيف & 7 \\
\hline & & ثانيا : العمل كفريق & \\
\hline 1,01 & $\varepsilon, r \mu$ & يعطي الفندق اولويـة للعمل كفريق & $\checkmark$ \\
\hline $1, r \cdot \varepsilon$ & $\varepsilon, \varepsilon$. & يشجـع الفندق العاملـين للعمل كفريق & $\Lambda$ \\
\hline $1, \cdots 7$ & $\varepsilon, 1 V$ & يعزز الفندق مشاركة العاملين يِّ اداء الخدمـة & 9 \\
\hline \multirow[t]{2}{*}{., 919} & $\varepsilon, \varepsilon$. & يكافىء الفندق فرق العهل ذات الاداء المتميز & 1. \\
\hline & & ثالثا : الثقافة التنظيمية & \\
\hline., 901 & $\varepsilon, r V$ & تتيح ثقافة الفندق تحسيـين الجودة بـاستمـرار & 11 \\
\hline., 910 & אוT & يتبنى الفندق ثقافة الجودة & ir \\
\hline $1, \cdots 1$ & r,qu & تركز قيهم الفندق على الاهتمام بـالعاملين & ir \\
\hline$\cdot$, V9V & $\varepsilon, r v$ & تشجـع ثقافة الفندق على اداء العمل مـن اول مرة & $1 \varepsilon$ \\
\hline 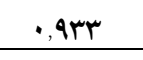 & $\varepsilon, r q$ & يسهل الفندق اتصال العـاملين ببعضهم البعض & 10 \\
\hline \multirow[t]{2}{*}{$\cdot, \wedge 70$} & $\varepsilon, 77$ & يعمل الفندق على تخفيض النزاعات الداخلية & 17 \\
\hline & & رابعا : الثقة المنظمية & \\
\hline$\cdot, 997$ & r,qV & يثق العاملون بـاداء الفندق & IV \\
\hline$\cdot$, VVo & $\varepsilon, r q$ & يشعر العاملون بالصدق يِ التعامل & M \\
\hline 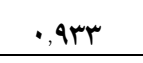 & $\varepsilon$, rq & هناك ثقة بين العاملـين انفسهم & 19 \\
\hline$\cdot, \wedge 77$ & $\varepsilon, 77$ & يلتزم الفندق بمعايير السلوك والاخلاق & r. \\
\hline
\end{tabular}

يشير الجدول رقم (ץ) بـان المتوسطات الحسابية لفقرات الاستبانة المتعلقـة بهـتغيرات الجـودة

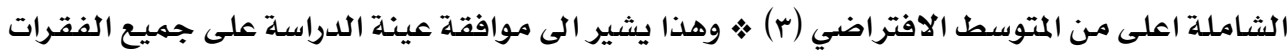

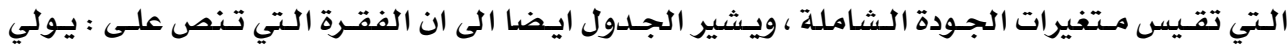




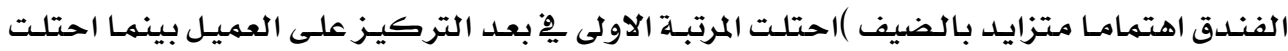

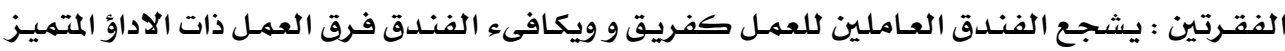

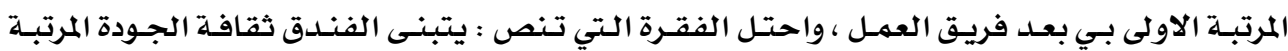

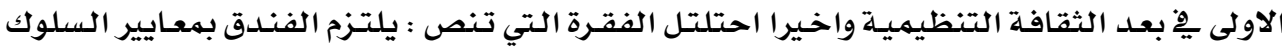
والاخلاق المرتبة الاولى يِّ بعد الثقدة المنظمية

\section{جدول رقم (r)}

المتوسطات الحسابية لرضا العاملين

\begin{tabular}{|c|c|}
\hline المتوسط الحسـابي & المتغير \\
\hline r,97 & التركيز على العميل \\
\hline$\varepsilon, r \wedge$ & العهل كفريق \\
\hline$\varepsilon, r q$ & الثقافة التنظيهية \\
\hline$\varepsilon, 7\}$ & الثقة \\
\hline
\end{tabular}

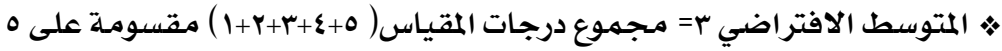
يتبـين مـن الجدول رقم (r) ان متغير الثقة جاء مِّف المرتبـة الاولى من متغيرات الجودة الشاملة

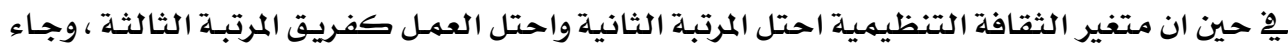

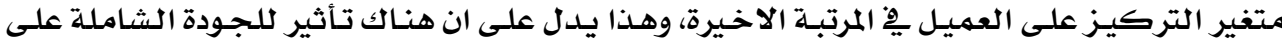

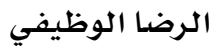

\section{اختبار فرضيات الدراسة}

الفرضية الرئيسية

توجد علاقة بين الجودة الشاملة يِ الفنادق والرضا الوظيفي

جدول رقم (ع)

قيمة اختبار (ت)، ومستوى دلالتها (قيم ألفا) للفرضية الرئيسية

\begin{tabular}{|c|c|c|c|c|}
\hline نتيـجـة الفرضيـة العدـميـة & $\mathrm{R}$ & T المعنوية & T الجدولية & T المحسوبة \\
\hline رفض & $\% 97, Y$ & $\cdot, \cdot r Y$ & 1,97 & $\varepsilon Y, \wedge T \Lambda$ \\
\hline
\end{tabular}

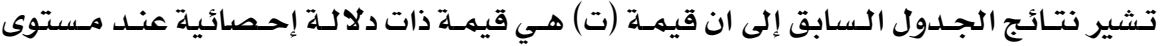

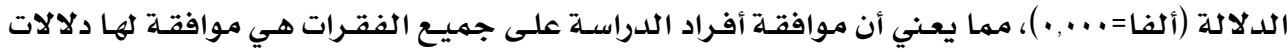

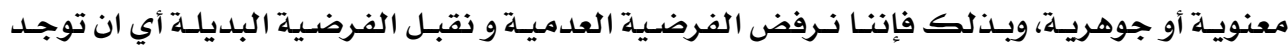

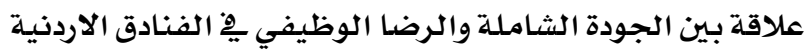




\section{• الفرضية الفرعية الأولى:}

توجد علاقة بين التركيز على العميل كأحد متغيرات الجودة الشاملـة والرضا الوظيفي هِ

$$
\text { الفنادق الاردنية }
$$

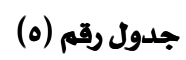

قيمة اختبار (ت)، ومستوى دلالتها (قيم ألفا) للمتغير المتعلق بالفرضية الفرعية الاولى

\begin{tabular}{|c|c|c|c|c|}
\hline نتيجـة الفرضيـة العدمية & $\mathrm{R}$ & المعنوية T & T الجدولية & T المحسوبة T \\
\hline رفض & $\% \wedge \vee, q$ & $\cdot, \cdots$ & 1,97 & $Y Y, \varepsilon V_{1}$ \\
\hline
\end{tabular}

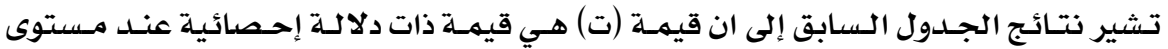

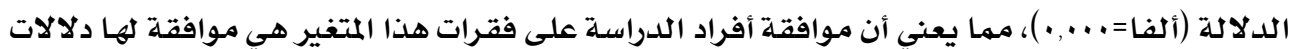

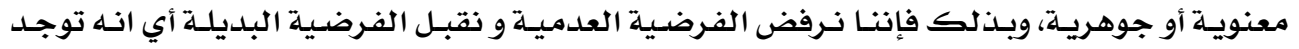

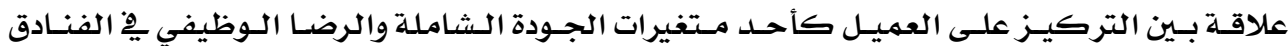
الاردنية وتعتبر هذه العلاقة قويةجلدا

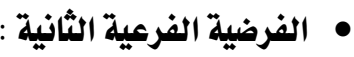

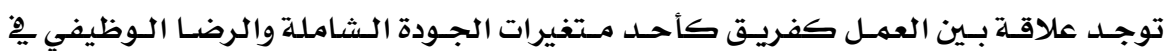

$$
\text { الفنادق الاردنيـة }
$$

\section{جدول رقم (7)}

\begin{tabular}{|c|c|c|c|c|}
\hline نتيجـة الفرضية العدمية & $\mathrm{R}$ & T المعنوية & الجدولية T & T المحسوبة T \\
\hline رفض & $\% \wedge 7,0$ & $\cdot, \cdots$ & 1,97 & $r \cdot 97$ \\
\hline
\end{tabular}

قيمة اختبار (ت)، ومستوى دلالتها (قيم ألفا) للمتغير المتعلق بالفرضية الفرعية الثانية

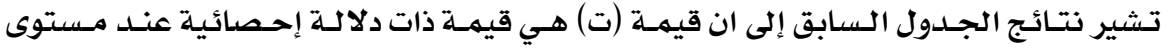

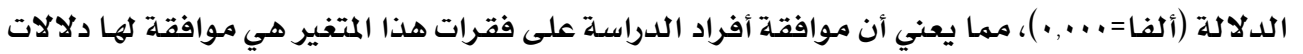

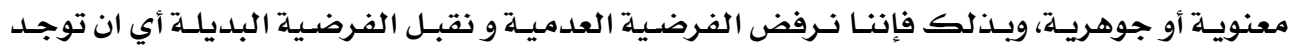

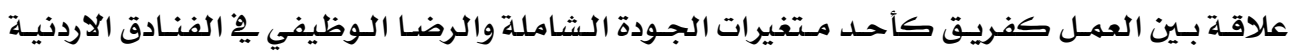
وتعتبر هذه العلاقة قوية • الفرضية الفرعية الثالثة:

توجد علاقة بـين الثقافة التنظيميـة كأحد مـتغيرات الجـودة الشاملة والرضـا الوظيفي ِِ

$$
\text { الفنادق الاردنية }
$$


جدول رقم (v)

قيمة اختبار (ت)، ومستوى دلالتها (قيم ألفا) للمتغير المتعلق بالفرضية الفرعية الثالثة

\begin{tabular}{|c|c|c|c|c|}
\hline نتيججة الفرضية العدمية & $\mathrm{R}$ & T المعنوية & T الجدولية & T المحسوبـة \\
\hline رفض & $\% 9 \wedge, Y$ & $\cdot, \cdots$ & 1,97 & ו ו וT, \\
\hline
\end{tabular}

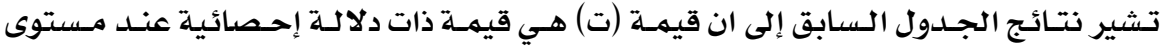

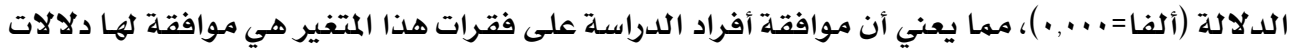

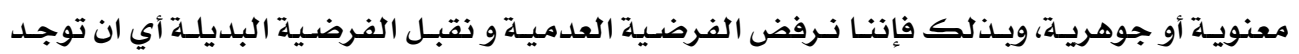

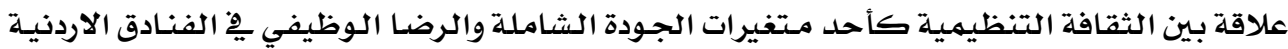
وتعتبر هذه العلاقة قوية

\section{• الفرضية الثرعية الرابعة:}

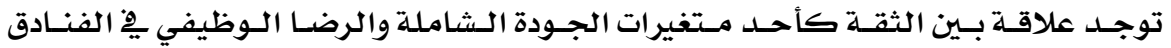
الاردنيـة

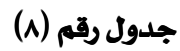

قيمة اختبار (ت)، ومستوى دلالتها (قيم ألفا) للمتغير المتعلق بالفرضية الفرعية الرابعة

\begin{tabular}{|c|c|c|c|c|}
\hline نتيججة الفرضية العدمية & $\mathrm{R}$ & المعنوية T & T الجدولية & المحسوبة T \\
\hline رفض & $\%, 94,0$ & $\cdot, \cdots$ & 1,97 & $r r, \cdot 1 \varepsilon$ \\
\hline
\end{tabular}

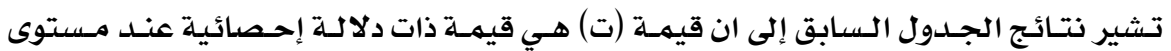

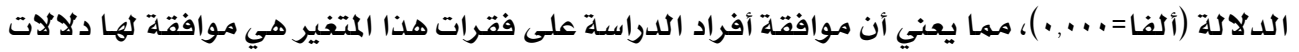

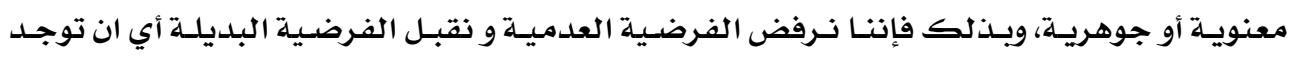

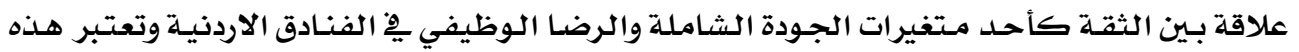
العلاقة قوية.

\section{النتائج والتوصيات}

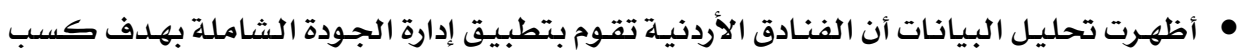

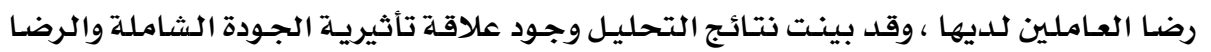

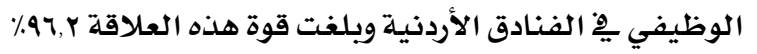

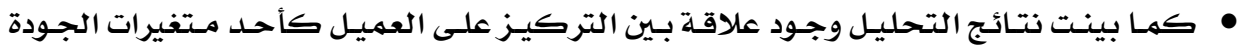

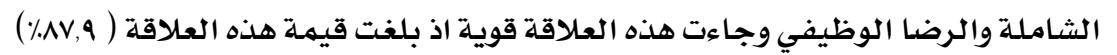




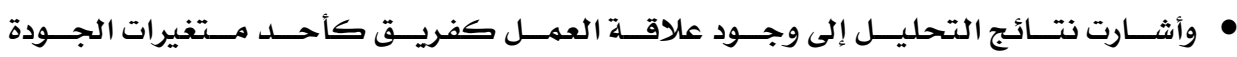

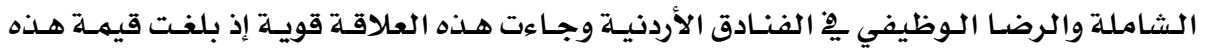

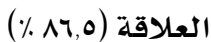

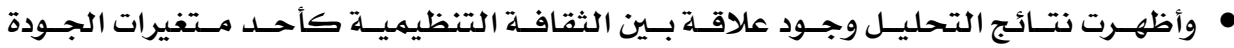

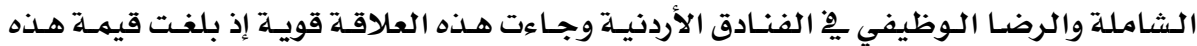

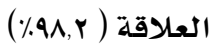

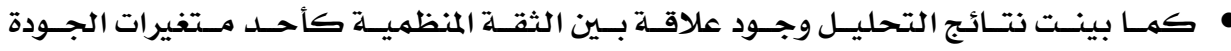

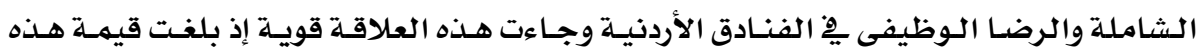

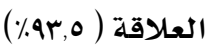

التتوصيات

ِِْ ضوء مناقشة النتائج تقترح الدراسة التوصيات التالية • أن تركز الفنـادق الأردنيـة على رضـا العـاملين لـديها إذ أن رضـاهم ترسيخ انتمـاؤهم وولاؤهـم

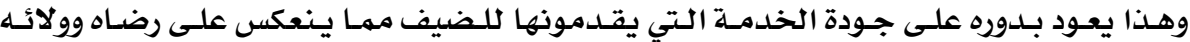
للفندق • ومن اجل تحقيق ذلك على الفنادق الأردنية أن تعمل على تطبيق إدارة الجـودة الشاملة بكافـة عناصرها

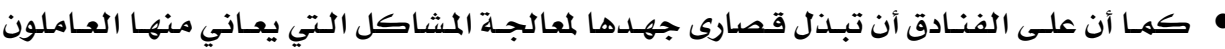
والاهتمام بها وتذليل الصعاب التي يواجهونها

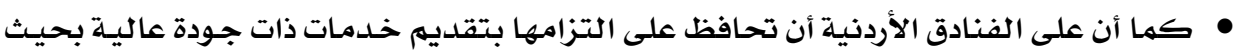
تلبي توقعات ورغبات الضيوف . 


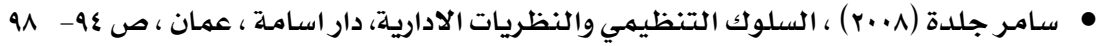

- Alina Ieana and rob Simmons ( 2006) Human Resources Management Practices and Worker's Job satisfaction, International journal of man power, Vol 29, no.7, pp 661-667

- Chang Lee, and Kley Way ( 2009), Individual Employment characteristics of Hotel employees that playa role in employee's satisfaction and work retention, International of Hospitality Management

- Teny Lam , Hanqin Zhang and Tom Baum ( 2001) An investigation of employees job satisfaction : the case of Hotels in Hong Kong, tourism Management , 22 , pp 157-165

- Belfield, C. and Harris, R. (2002), "How well do theories of job matching explain variation in job satisfaction across educational level? Evidence for UK graduates", Applied Economics,Vol. 34 No. 5, pp. 535-48.

- Boselie, P. and Van der Wiele, T. (2002), "Employee perceptions of HRM and TQM, and the effects on satisfaction and intention to leave", Managing Service Quality, Vol. 12 No. 3,pp. 165-72.

- Clark, A.E. (2005), "Your money or your life: changing job quality in OECD countries", Working Paper 1610, Institute for the Study of Labor (IZA), Bonn.

- Clark, A.E. and Oswald, A.J. (1996), "Satisfaction and comparison income", Journal of Public Economics, Vol. 61 No. 3, pp. 359-81.

- Luchak, A.A. (2003), "What kind of voice do loyal employees use?", British Journal of Industrial Relations, Vol. 41 No. 1, pp. 115-34.

- Oswald, A. (1997), "Happiness and economic performance", The Economic Journal, Vol. 107No. 445, pp. 1815-31.

- Keng Boon Ooi, Nooh Abu Bakar, Veeri Arumugam ,Lorraine Vellapan and Alex Kim Yin Loke (2007) Does TQM influence employees' job satisfaction? An empirical case analysis ,International Journal of Quality \&Reliability Management, Vol. 24 No. 1 pp. 62-77 
- Haccoun, R. and Jeanrie, C. (1995), "Self-reports of work absence as a function of personal attitudes toward absence, and perceptions of the organization", applied Psychology: An International Review, Vol. 44, and pp. 155-70.

- Berry, L. (1997), Psychology at Work, McGraw-Hill, New York, NY.

- Layard, R. (2006), "Happiness and public policy: a challenge to the profession", Economic Journal, Vol. 116 No. 510, pp. C24-C33.

- Alina Ieana and Rob Simmons (2006) Human Resources Management Practices and Worker's Job satisfaction, International journal of man power, Vol 29, no.7, pp 661-667

- Teny Lam , Hanqin Zhang and Tom Baum ( 2001) An investigation of employees job satisfaction : the case of Hotels in Hong Kong, tourism Management , 22 , pp 157-165

- Beatty, C. (1996), "The stress of managerial and professional women: is the price too high?" Journal of Organizational Behavior, Vol. 17, pp. 233-51.

- Zhang, Z.H. (2000), "Implementation of total quality management: an empirical study of Chinese manufacturing firms", $\mathrm{PhD}$ unpublished thesis, University of Groningen, Groningen

- Oakland, J.S. and Oakland, S. (2001), "Current people management activities in world-class organizations”, Total Quality Management, Vol. 12 No. 6, p. 773.

- Noorliza, K. and Zainal, A.A. (2000), "Quality practices that pay: empowerment and teamwork", Malaysian Management Review, Vol. 35 No. 2, pp. 66-76.

- Gray, J.H., Densten, I.L. and Sarros, J.C. (2003), "A matter of size: does organizational culture predict satisfaction in small organizations?", working paper 65/03, September, Faculty of Business and Economics, Monash University, Australia

- Alf Crossman and Bassem Abou-Zaki ( 2006), Job satisfaction and employee performance of Lebanese banking staff, Journal of Managerial Psychology Vol. 18 No. 4, 2003 pp. 368-376 
- Oswald, A. and Gardner, J. (2001), "What has been happening to the quality of worker's lives in Britain?", working paper, Department of Economics, University of Warwick, Coventry.

- Cruickshank, M.T. (2000), "Developing a quality culture within a school of nursing in higher education", PhD unpublished thesis, University of Western Sydney, Hawkesbury.

- Daulatram B. Lund( 2003), Organizational culture and job satisfaction

- JOURNAL OF BUSINESS \& INDUSTRIAL MARKETING, VOL. 18 NO.3 2003, pp. 219-236.

- Lok, P. and Crawford, J. (1999), "The relationship between commitment and organizational culture, subculture, leadership style and job satisfaction in organizational change and development", Leadership and Organizational Development Journal, Vol. 20, pp. 365-73.

- Cramer, D. (1996), "Job satisfaction and organizational continuance commitment: a two-wave panel study", Journal of Organizational Behavior, Vol. 17, pp. 389-400.

- Currivan, D.E. (1999), "The causal order of job satisfaction and organizational commitment in models of employee turnover", Human Resource Management Review, Vol. 9, pp. 495-524. 